\title{
Flight tone characterisation of the South American malaria vector Anopheles darlingi (Diptera: Culicidae)
}

\author{
Jose Pablo Montoya', Hoover Pantoja-Sánchez',3, Sebastian Gomez ${ }^{1,2}$, \\ Frank William Avila ${ }^{4}$, Catalina Alfonso-Parra ${ }^{1,4} /+$ \\ ${ }^{1}$ Universidad CES, Instituto Colombiano de Medicina Tropical, Sabaneta, Antioquia, Colombia \\ ${ }^{2}$ Universidad de Antioquia, Departamento de Ingeniería Electrónica, Medellín, Antioquia, Colombia \\ ${ }^{3}$ Universidad de Antioquia, Programa de Estudio y Control de Enfermedades Tropicales, Medellín, Antioquia, Colombia \\ ${ }^{4}$ Universidad de Antioquia, Max Planck Tandem Group in Mosquito Reproductive Biology, Medellín, Antioquia, Colombia
}

BACKGROUND Flight tones play important roles in mosquito reproduction. Several mosquito species utilise flight tones for mate localisation and attraction. Typically, the female wingbeat frequency (WBF) is lower than males, and stereotypic acoustic behaviors are instrumental for successful copulation. Mosquito WBFs are usually an important species characteristic, with female flight tones used as male attractants in surveillance traps for species identification. Anopheles darlingi is an important Latin American malaria vector, but we know little about its mating behaviors.

OBJECTIVES We characterised An. darlingi WBFs and examined male acoustic responses to immobilised females.

METHODS Tethered and free flying male and female An. darlingi were recorded individually to determine their WBF distributions. Male-female acoustic interactions were analysed using tethered females and free flying males.

FINDINGS Contrary to most mosquito species, An. darlingi females are smaller than males. However, the male's WBF is $\sim 1.5$ times higher than the females, a common ratio in species with larger females. When in proximity to a female, males displayed rapid frequency modulations that decreased upon genitalia engagement. Tethered females also modulated their frequency upon male approach, being distinct if the interaction ended in copulation or only contact.

MAIN CONCLUSIONS This is the first report of An. darlingi flight acoustics, showing that its precopulatory acoustics are similar to other mosquitoes despite the uncommon male:female size ratio, suggesting that WBF ratios are common communication strategies rather than a physical constraint imposed by size.

Key words: flight acoustics - reproduction - wing beat - flight-tone - malaria

Malaria is one of the most important vector-borne diseases worldwide, with 219 million cases reported in 2017. (1) In South America, Anopheles darlingi is a major malaria vector, ${ }^{(2,3)}$ being the primary neotropical malaria vector in the Amazon region of Brazil, Colombia, Peru and Venezuela. ${ }^{(4)} A n$. darlingi is an efficient malaria vector, able to maintain high levels of transmission even when present at low densities. ${ }^{(3)}$ Further, genetic differentiation among An. darlingi populations is suggested to enable adaptation of this species to a range of habitats. ${ }^{(4,5)}$ However, little is known about the basic biology of this species, and its reproductive behaviors have not been reported to date, making the control of this vector challenging.

Disruption of pre-mating reproductive behaviors has been proposed as a means to control mosquito populations by exploiting important mating-specific cues to prevent male-female interaction. ${ }^{(6,7)}$ One area of focus is precopulatory behavioral interactions between males

doi: 10.1590/0074-02760200497

Financial support: COLCIENCIAS [Grant CD 325671250804 (CT 409-2016) (to CAP)] and COLCIENCIAS, Universidad de Antioquia and the Max

Planck Society cooperation [Grant 566-1-2014 (to FWA)].

JPM and HP-S contributed equally to this work.

+ Corresponding author: catalfonso@gmail.com

(D) https://orcid.org/0000-0002-5689-9356

Received 24 September 2020

Accepted 23 February 2021 and females. Prior to copulation, male and female mosquitoes must locate each other and interact. Males are attracted to tones produced by the female wing beat, ${ }^{(8,9)}$ although additional cues are likely to aid male-female attraction. $^{(10)}$ The mating encounter site for some species is around the host (e.g., Aedes aegypti) - males intercept females as they attempt to blood-feed. ${ }^{(11,12)}$ Males of some anopheline species form swarms - females penetrate the swarm to find a mate; (13) it is unknown if $A n$. darlingi males form swarms.

Male and female Ae. aegypti, Anopheles gambiae, Anopheles albimanus and Culex quinquefasciatus interact acoustically pre-copula. ${ }^{(14,15,16,17,18)}$ One such interaction is rapid frequency modulation (RFM), the rapid increase of the male wing beat frequency (WBF), followed by tone oscillation, terminating with a decrease in tone frequency. $(19,20)$ This phenomenon occurs when males approach females during a mating attempt ${ }^{(19,20)}$ and appears to be a common male acoustic behavior during courtship, having been described in Ae. aegypti, Cx. quinquefasciatus, An. gambiae, An. coluzzi, and An. albimanus. ${ }^{(17,19,20,21)}$ Further, in Ae. aegypti, Cx. quinquefasciatus, and An. gambiae males and females modulate their WBFs to match in a shared harmonic during courtship, a phenomenon known as harmonic convergence. ${ }^{(14,16,18,22)}$

Mosquito WBFs can be an important characteristic of a species, ${ }^{(23,24,25)}$ as it mediates mating, although closely related species can have similar flight tones. ${ }^{(10)}$ In addi- 
tion, WBFs are influenced by factors such as temperature, humidity and age. ${ }^{(22,26)}$ Intraspecific body size has also been suggested to influence $\mathrm{WBF},{ }^{(22,27)}$ although other reports find no such effect. ${ }^{(17,28)}$ Thus, how body size influences flight tones of individuals remains unclear. In addition, males and females of most species usually exhibit different wing-beat frequencies, with males producing higher frequencies. It has long been assumed that this frequency difference is associated with the smaller size of males. ${ }^{(9,12,29)}$ However, in An. darlingi, males and females collected in the field are similarly sized. ${ }^{(30)}$ This species therefore presents an ideal opportunity to evaluate how male-female size ratio influences mosquito acoustic interactions. Furthermore, evaluation of the acoustic mating behaviors of An. darlingi, an understudied species, will contribute to our knowledge of mosquito mating behaviors.

To examine precopulatory behavioral interactions in an understudied malaria vector, we characterised $A n$. darling $i \mathrm{WBF}$ and examined acoustic behaviors upon exposure to the opposite sex. We find that lab reared males are larger than females, unlike other mosquito species. Although larger, males broadcast a significantly higher WBF than females, showing that the WBF ratio between males and females is not determined by body size, contrary of what has been assumed in the past. ${ }^{(9,12,29)}$ Upon exposure to tethered females, males displayed RFM upon female approach. This behavior is observed in other mosquito species, showing that the communication dynamic during mating is preserved in An. darlingi despite the particular size of males and females of this species. These findings support the idea that the acoustic dynamic of mosquito mating behavior is a process of communication rather than a byproduct of motion. Moreover, as flight tones are currently being used in the development of novel surveillance strategies, ${ }^{(6,23,25,31-33)}$ insight into $A n$. darlingi reproductive behaviors and WBF characterisation will aid their surveillance and control.

\section{MATERIALS AND METHODS}

Mosquitoes - An. darlingi from Universidad Peruana Cayetano Heredia - ICEMR insectary (Iquitos, Peru) were used in our experiments. This colony has been maintained since 2012. ${ }^{(34,35)}$ Pupae were individualised in $5 \mathrm{~mL}$ tubes to ensure virginity and adults were separated by sex and transferred to sex-specific cages upon eclosion. Mosquitoes had access to $15 \%$ honey-water solution ad libitum. All recordings were conducted at $26^{\circ} \mathrm{C}$ and $80 \%$ relative humidity (RH). Five to seven-day-old mosquitoes were used in all experiments.

Audio recording set up - Mosquitoes were recorded in a $4 \mathrm{~L}$ plastic cage using a particle velocity microphone (NR-23158-000, Knowles; Itasca, IL, USA). A USB audio interface (M-Track Quad Four Channel Audio; M-Audio, Cumberland, USA) was used to amplify and digitise recordings at a sample rate of $11025 \mathrm{~Hz} / 24$ bits.

Experimental procedures - We first recorded mosquitoes individually to determine WBFs when in free flight and when tethered. As similar aged individuals were difficult to obtain from this colony (i.e., we could not synchronise hatch rates and experienced high mortality subsequent to individualisation), the same mosquitoes were utilised to determine WBF under both conditions. Tethered mosquitoes were immobilised as in Pantoja-Sánchez et al. ${ }^{(17)}$ and placed $1 \mathrm{~cm}$ above the microphone to record their WBFs. Free flying mosquitoes were recorded using a rod with an adhered microphone; a researcher manually followed their flight, maintaining a distance of 5-10 $\mathrm{cm}$. We next recorded free flying males and tethered females to examine acoustic interactions during a mating interaction - an immobilised female was placed $\sim 5 \mathrm{~cm}$ from the microphone to allow movement around her [Supplementary data (Figure)]. Ten-fifteen males were then introduced into the cage. Females were replaced upon copulation; spermathecae were subsequently dissected to determine insemination status. We recorded all trials with a camera (FLIR-FLEA 3 1.3 MPColor USB 3 Vision with a Fujinon - FF125HA1B $12.5 \mathrm{~mm}$ lens) and identified and timed behaviors in real time. Mosquito wings were measured as in van den Heuvel et al. ${ }^{(36)}$ to estimate body size.

Signal and statistical analysis - Flight-tone audio recordings were analysed using spectrograms (Fast Fourier transform-based, length of 4096 points, hamming window of $80 \mathrm{~ms}$ and $50 \%$ overlapping). To evaluate acoustic interactions, audio segments occurring during observed mating attempts were analysed. From the spectrograms, we used the male second harmonic and the female third to examine flight-tones during malefemale behavioral interactions due to frequency resolution but present our results in terms of their fundamental WBFs for simplicity, as done previously. ${ }^{(17)}$ We report the time of the interaction as the length of male's flight tone. Male and female responses were assessed by evaluating the extent of frequency modulation in the second and third harmonic, respectively. Male measurements were divided by two $\left(\Delta \mathrm{F}=\left(\mathrm{F}_{\max }-\mathrm{F}_{\min }\right) / 2\right)$ and female measurements by tree $\left(\Delta \mathrm{F}=\left(\mathrm{F}_{\max }^{\max }-\mathrm{F}_{\text {min }}^{\min }\right) / 3\right)$ to express results in terms of fundamental frequency equivalent to the WBF. We further assessed female behavior by examining the rate of increase $(\Delta \mathrm{F} / \Delta \mathrm{t})$ after male detection.

To compare frequencies between flight conditions (tethered and free flight), and to discriminate differences between sexes, we used t-tests. Normality for the variable WBF was determined with a Shapiro-Wilk test. A linear regression was used to determine the relationship between wing size and WBF. Residuals were tested for normality, homogeneity of variance and independence using Shapiro-Wilks, Bartlett and Durbin-Watson tests, respectively. We compared male-female contact and copulation interactions using the non-parametric MannWhitney $U$ test to assess similarities and differences in the distributions of the variables analysed. Results are reported as mean \pm standard deviation for variables that followed a normal distribution, and as median (interquartile range - IQR) for variables that did not. Signal processing was performed using Matlab (R2016a, Mathworks Inc., Natick, USA). Statistical analysis was performed using the car package ${ }^{(37)}$ of R (Vienna, Austria). ${ }^{(38)}$ 


\section{RESULTS}

Body sizes of An. darlingi adults - Using dry weight to examine body size in field collected An. darlingi, Lounibos et al. ${ }^{(30)}$ reported that males and females of this species were similarly sized. As An. darlingi dry weight is highly correlated with wing length, ${ }^{(30)}$ we measured wing lengths to determine male and female size of our lab reared specimens. Female An. darlingi $(2518 \pm 88$ $\mu \mathrm{m})$ were significantly smaller than males $(2635 \pm 14$ $\mu \mathrm{m})$ (t-test: $\mathrm{t}_{(29)}=3.82, \mathrm{p}<0.01$; Fig. 1A) when reared under standard laboratory conditions unlike what has been observed in many species across different genera of Culicidae. ${ }^{(9,12)}$ The male/female size ratio in An. darlingi (size ratio 1.046) is distinct compared to other species in the same genus such as An. albimanus (size ratio: $0.95)^{(17)}$ or in a different genus such as Ae. aegypti (size ratio: 0.81$)^{(39)}$ (Fig 1A).

Wing beat frequencies of male and female An. Darling - We recorded individual adults to characterise their flight tones under two conditions: when tethered and in free flight as some reports suggest that tethering impacts WBFs while others report no affect. ${ }^{(17,19,28,40)}$ We found significant differences between the two conditions: the mean WBF of tethered males was $553 \pm 78$ $\mathrm{Hz}(\mathrm{n}=30)$, and $709 \pm 42 \mathrm{~Hz}(\mathrm{n}=30)$ in free flight $(\mathrm{t}-$ test: $\left.\mathrm{t}_{(29)}=-9.36, \mathrm{p}<0.01\right)$. The mean WBF of tethered females was $362 \pm 33 \mathrm{~Hz}(\mathrm{n}=30)$, and $456 \pm 33 \mathrm{~Hz}(\mathrm{n}=$ 30 ) in free flight (t-test: $t_{(29)}=-10.97, p<0.01$; Fig. 1B). Although males are larger than females (Fig. 1A), males broadcast significantly higher frequencies (1.53 times higher) than females when tethered (t-test: $\mathrm{t}_{(29)}=12.31$, $\mathrm{p}<0.01)$ and 1.55 times higher in free flight ( $\mathrm{t}$-test: $\mathrm{t}_{(29)}$ $=26.10, \mathrm{p}<0.01)$ similar to what has been observed in other species such as Ae. aegypti and An. albimanus. ${ }^{(17,40)}$ Within each sex, we did not detect an effect of size on the WBF when tethered (Linear regression: males: $\mathrm{R}^{2}=0.071, \mathrm{~F}_{28}=2.14, \mathrm{t}=-1.462, \mathrm{p}=0.1549$; females: $\mathrm{R}^{2}$ $\left.=0.001, \mathrm{~F}_{28}=0.055, \mathrm{t}=0.23427, \mathrm{p}=0.8165\right)$.
Male-female acoustic interactions during a mating attempt - To investigate acoustic interactions between sexes, we exposed free flying males to a tethered-flying female. We analysed all interactions where the male's flight-tone was detected. Two types of interactions were observed: (1) male-female leg contact and (2) copulation, defined as visible genitalia engagement. However, sperm transfer was never detected upon female dissection, possibly due to the inability to achieve a proper angle for insemination. ${ }^{(41)}$

Male signal was detected from 40 distinct interactions using 15 females (11 copulations, 29 contacts). In all interactions, when nearing the female, RFM of the male flight tone occurred followed by modulation of the female frequency (Fig. 2). In copulation interactions, the median time of male interaction was $2.35 \mathrm{~s}(\mathrm{n}=11$, IQR 1.95 - $4.82 \mathrm{~s}$ ), male flight-tones were characterised by RFMs with a modulation extent $\left(\mathrm{F}_{\max }-\mathrm{F}_{\min }\right)$ of 396.51 $\mathrm{Hz}$ (IQR 296.84 - 460.69 Hz). RFM was followed by a decrease in the modulation (Fig. 2A) or by male wing beat cessation; in both cases while engaged with the female genitalia. In contact interactions, the median time of male interaction was $2.36 \mathrm{~s}(\mathrm{n}=29$, IQR $2.13-3.78$ s), male flight-tones were characterised by RFMs until departure from the female (Fig. 2B), with a modulation extent of $321.99 \mathrm{~Hz}$ (IQR 279.347 - $383.14 \mathrm{~Hz}$ ). No differences in the interaction time (Mann-Whitney U-test: $\mathrm{U}_{11,29}=129.00, \mathrm{Z}=0.92, \mathrm{p}=0.35$; Fig. 3A) or modulation extent (Mann-Whitney U-test: $U_{11,29}=113.00, Z=-1.41, p$ $=0.16$; Fig. 3B) were detected between interaction types.

Female flight-tones were also analysed. From the 40 interactions described above, 12 were excluded from our analysis as females stopped their wing beat upon male contact. Despite being tethered, female signal modulation was detected in the remaining 28 interactions (11 copulations, 17 contacts). Females reacted to the male approach by modulating their flight-tone frequency — we observed an initial WBF increase similar to descriptions in other mosquito species..$^{(19,21)}$ The magnitude of the frequency increase in contact (median $130.38 \mathrm{~Hz}$, IQR 73.18
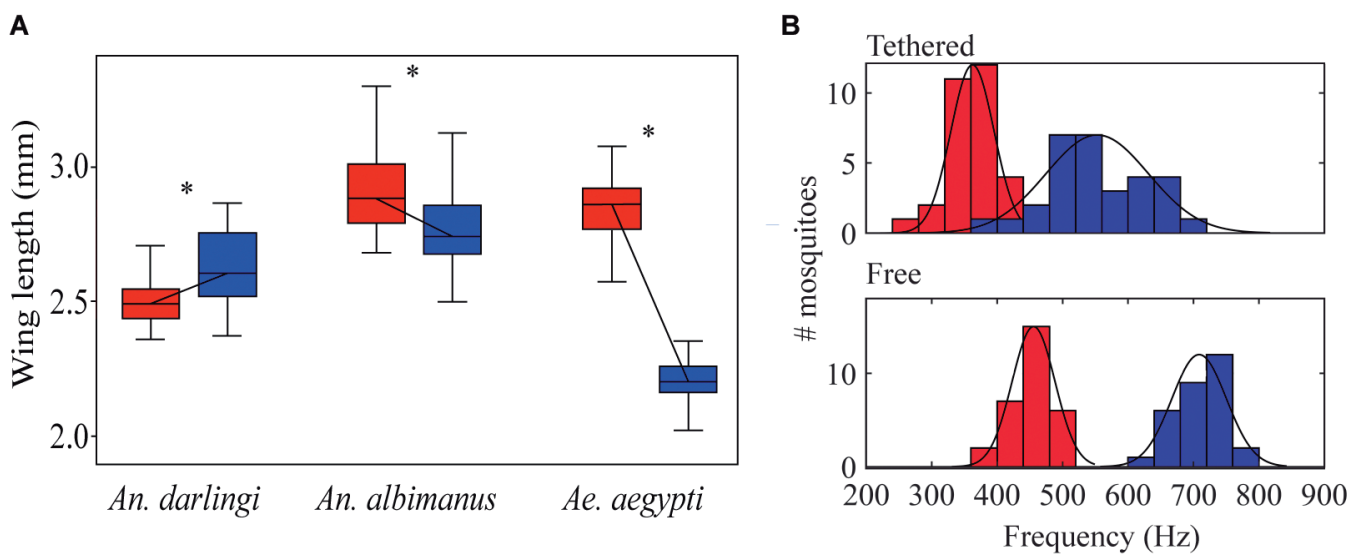

Fig. 1: wing beat frequencies and sizes of male and female Anopheles darlingi. We used wing length as a proxy for body size. (A) Distribution of female (red; $\mathrm{n}=30$ ) and male size (blue; $\mathrm{n}=30$ ). Sizes for male and female Aedes aegypti ${ }^{(39)}$ and An. albimanus ${ }^{(17)}$ are shown for comparison. (B) Top panel: frequency distribution of tethered females (red; $\mathrm{n}=30$ ) and males (blue; $\mathrm{n}=30)$. Bottom panel: frequency distribution of free-flying females (red; $\mathrm{n}=30$ ) and males (blue: $\mathrm{n}=30$ ). 
A

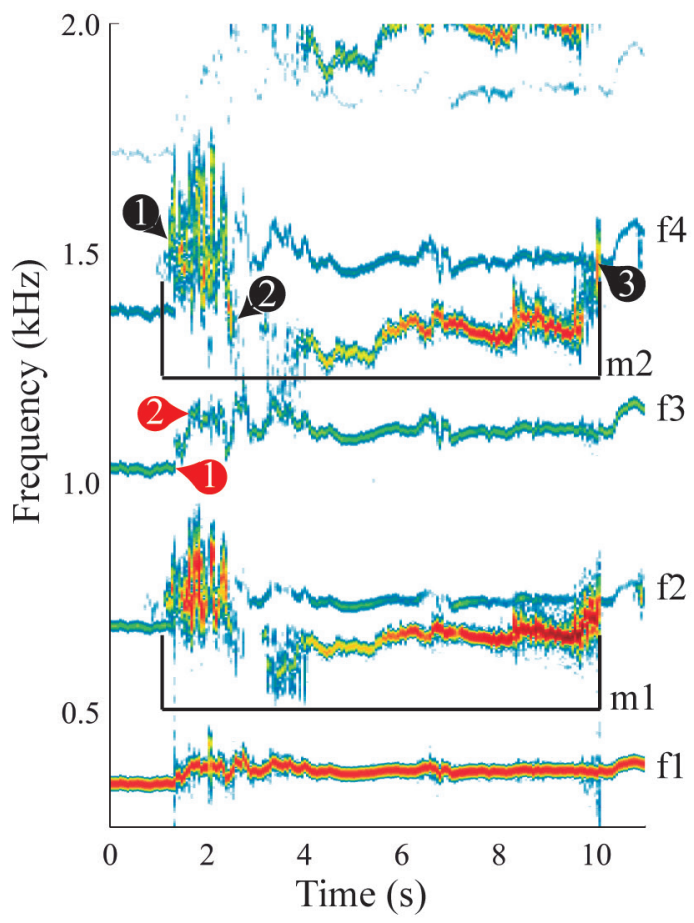

B

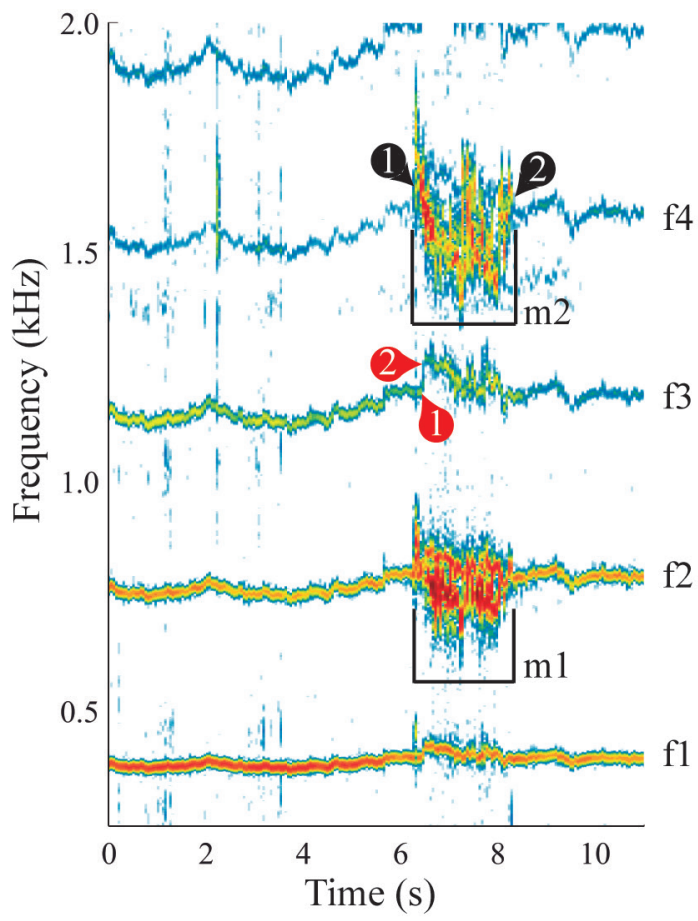

Fig. 2: male-female spectrograms in interactions that end in copulation (A) or only contact (B). Female (f) and male (m) fundamental frequency and its harmonics are shown. Colours indicate the power of each frequency component; red is the most powerful, blue the least powerful, and white indicates the noise floor. Notable events are indicated for males (black numbers) and females (red); male signal is specified with a bracket in (A) and (B). (A) Male rapid frequency modulation (RFM) begins (black 1) and ends (black 2). In this interaction, male flight tone was stable until disengagement from the female (black 3). (B) RFM begins (black 1) and ends (black 2), with male departure shortly afterwards. In both outcomes, the female increased her wing beat frequency (WBF) upon male detection (red 1), quickly reaching peak frequency (red 2).

- $167.72 \mathrm{~Hz}$ ) and copulation interactions (median 101.78 $\mathrm{Hz}$, IQR $75.19-144.87 \mathrm{~Hz}$ ) was similar (Mann-Whitney U-test: $\mathrm{U}_{11,17}=83, \mathrm{Z}=0.49, \mathrm{p}=0.62$; Fig. 3C). However, the rate of frequency increase in contact interactions (median $158.46 \mathrm{~Hz} / \mathrm{s}$, IQR 117.86 - $304.40 \mathrm{~Hz} / \mathrm{s}$ ) was significantly higher compared to copulation interactions (median $112.67 \mathrm{~Hz} / \mathrm{s}$, IQR $75.99-159.94 \mathrm{~Hz} / \mathrm{s}$; Mann-Whitney U-test: $U_{11,17}=47, Z=2.18, p=0.028$; Fig. 3D).

\section{DISCUSSION}

Mosquito mating has been widely used as a target to develop novel control strategies. ${ }^{(42)}$ The effectiveness of such strategies, however, require a profound understanding of behaviors associated with mosquito reproduction, ${ }^{(42)}$ in which flight-tones play a major role. ${ }^{(21,43)}$ Increasing our knowledge on the mating behavior of various species provides new insights on different aspects of acoustic interactions that influence mating. This study investigates the mating behavior of a particular mosquito species: $A n$. darlingi. We show how An. darlingi bioacoustics are similar to other species despite their uncommon intraspecific male/female size ratio..$^{(9,12,29)}$

When exposed to a tethered female, male RFM occurred when approaching and contacting the female. Similar observations in Cx. quinquefasciatus, An. gambiae, An. coluzzi and Ae. aegypti, indicate the importance of this behavior. ${ }^{(19,20,21)}$ RFM duration was similar in all interactions but varied greatly during male-female contact in the absence of copulation. In Ae. aegypti, RFM cessation coincides with formation of the ventralventral copulation position. ${ }^{(21)}$ Similarly, An. darlingi RFM ends when a pair engage their genitalia; the male decreases the frequency modulation of his tone or ceases flying. This might be a general Anopheline behavior, as mating pairs fall to the ground after couple formation. (44,45) Tethered An. darlingi females also modulated their flight tone. When males neared or made contact, tethered females increased their WBF at a higher rate than when the interaction resulted in copulation. This might be reflective of active female acceptance or rejection, although more experiments are necessary to test this hypothesis. Tethered Culex females modulate their WBF as a result of physical contact by the male. ${ }^{(19)}$ Whether WBF modulation by $A n$. darlingi females initiates during the male approach or only upon physical contact will require higher resolution videos.

Contrary to what has long been assumed, ${ }^{(9,12,29)}$ this study shows that male-female WBF distributions of medically relevant mosquito species and acoustic interactions that occur in mating attempts are not constrained exclusively by the intraspecific male/female size ratio. Moreover, our findings support the hypothesis that the characteristics of the acoustic dynamic between males and females correspond to a communication strategy rather than a byproduct of motion. By describing the precopulatory acoustic behaviors and WBF distribu- 
A

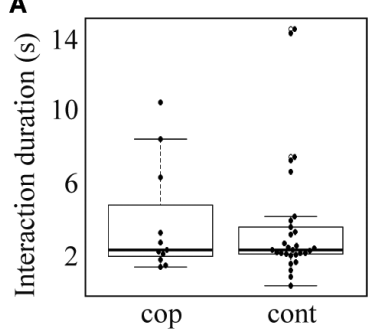

C

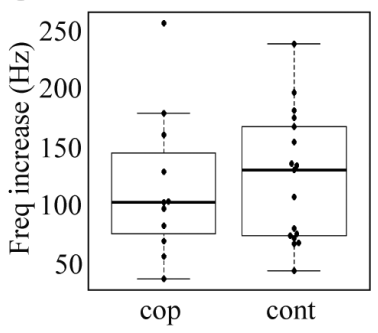

B

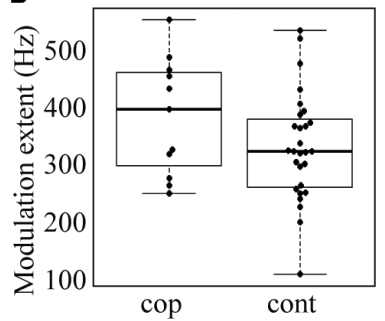

D

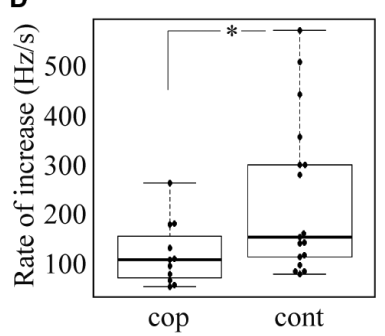

Fig. 3: comparison between copulation (cop) and contact (cont) interaction duration (A), modulation range of male flight tone (B), frequency increase of female flight tone $(C)$, and rate of frequency increase in female flight tone (D). Only the female rate of frequency increase significantly differed between interaction types, indicated by $*(p<0.05)$. Boxplots indicate the minimum, maximum, median, first quartile and third quartile in the data set.

tions of An. darlingi, this study contributes to the overall knowledge of the flight acoustics of this vector and mosquitoes in general. We hope this study will promote future investigations on species of medical relevance for Latin America and aid their surveillance.

\section{ACKNOWLEDGEMENTS}

To members of the Universidad Peruana Cayetano Heredia - ICEMR for providing the mosquitoes used in our experiments, with special thanks to Manuela Herrera, and Talya Shragai for helpful comments on the manuscript.

\section{AUTHORS' CONTRIBUTION}

Conception and design - HPS, SG and CAP; development of methodology - HPS, SG and CAP; data acquisition - SG and CAP; analysis and interpretation of data - JPM, HPS, SG, FWA and CAP; writing and review of the manuscript - JPM, HPS, FWA and CAP. All authors reviewed and approved the final manuscript.

\section{REFERENCES}

1. WHO - World Health Organization. WHO Report. World Malaria Report 2017. Available from: https://www.mmv.org/sites/default/ files/uploads/docs/publications/WMR2017.pdf.

2. Sinka ME, Bangs MJ, Manguin S, Rubio-Palis Y, Chareonviriyaphap T, Coetzee M, et al. A global map of dominant malaria vectors. Parasit Vectors. 2012; 5: 69.

3. Hiwat H, Bretas G. Ecology of Anopheles darlingi Root with respect to vector importance: a review. Parasit Vectors. 2011; 4: 177.

4. Chu VM, Sallum MAM, Moore TE, Lainhart W, Schlichting CD, Conn JE. Regional variation in life history traits and plastic responses to temperature of the major malaria vector Nyssorhynchus darlingi in Brazil. Sci Rep. 2019; 9: 5356.
5. Campos M, Conn JE, Alonso DP, Vinetz JM, Emerson KJ, Ribolla PEM Microgeographical structure in the major neotropical malaria vector Anopheles darlingi using microsatellites and SNP markers. Parasit Vectors. 2017; 10: 76.

6. Diabate A, Tripet F. Targeting male mosquito mating behaviour for malaria control. Parasit Vectors. 2015; 8: 347.

7. Andrés M, Su MP, Albert J, Cator LJ. Buzzkill: targeting the mosquito auditory system. Curr Opin Insect Sci. 2020; 40: 11-7.

8. Charlwood JD, Jones MDR. Mating behaviour in the mosquito, Anopheles gambiae s.1.save. Physiol Entomol. 1979; 4(2): 111-20.

9. Belton P. Attraction of male mosquitoes to sound. J Am Mosq Control Assoc. 1994; 10(22): 297-301.

10. Tripet F, Dolo G, Traoré S, Lanzaro GC. The "wingbeat hypothesis" of reproductive isolation between members of the Anopheles gambiae complex (Diptera: Culicidae) does not fly . J Med Entomol. 2004; 41(3): 375-84.

11. Yuval B. Mating systems of blood-feeding flies. Annu Rev Entomol. 2006; 51: 413-40.

12. Clements AN. The biology of mosquitoes: sensory reception and behaviour. Vol. 2. UK: Chapman \& Hall; 1992.

13. Howell PI, Knols BGJ. Male mating biology. Malar J. 2009; 8: 1-10.

14. Cator LJ, Arthur BJ, Harrington LC, Hoy RR. Harmonic convergence in the love songs of the dengue vector mosquito. Science. 2009; 323(5917): 1077-9.

15. Gibson G, Warren B, Russell IJ. Humming in tune: sex and species recognition by mosquitoes on the wing. J Assoc Res Otolaryngol. 2010; 11(4): 527-40.

16. Pennetier C, Warren B, Dabiré KR, Russell IJ, Gibson G. "Singing on the wing" as a mechanism for species recognition in the malarial mosquito Anopheles gambiae. Curr Biol. 2010; 20(2): 131-6.

17. Pantoja-Sánchez H, Gomez S, Velez V, Avila FW, Alfonso-Parra C. Precopulatory acoustic interactions of the New World malaria vector Anopheles albimanus (Diptera: Culicidae). Parasit Vectors. 2019; 12: 386.

18. Warren B, Gibson G, Russell IJ. Sex recognition through midflight mating duets in Culex mosquitoes is mediated by acoustic distortion. Curr Biol. 2009; 19(6): 485-91.

19. Simões PMV, Ingham RA, Gibson G, Russell IJ. A role for acoustic distortion in novel rapid frequency modulation behaviour in free-flying male mosquitoes. J Exp Biol. 2016; 219: 2039-47.

20. Simões PMV, Gibson G, Russell IJ. Pre-copula acoustic behaviour of males in the malarial mosquitoes Anopheles coluzzii and Anopheles gambiae does not contribute to reproductive isolation. J Exp Biol. 2017; 220: 379-85.

21. Aldersley A, Cator LJ. Female resistance and harmonic convergence influence male mating success in Aedes aegypti. Sci Rep. 2019; 9: 2145.

22. Cator LJ, Ng'Habi KR, Hoy RR, Harrington LC. Sizing up a mate: variation in production and response to acoustic signals in Anopheles gambiae. Behav Ecol. 2010; 21(5): 1033-9.

23. Ouyang TH, Yang EC, Jiang JA, Lin TT. Mosquito vector monitoring system based on optical wingbeat classification. Comput Electron Agric. 2015; 118: 47-55.

24. Mukundarajan H, Hol FJH, Castillo EA, Newby C, Prakash M. Using mobile phones as acoustic sensors for high-throughput mosquito surveillance. Elife. 2017; 6: e27854.

25. Genoud AP, Basistyy R, Williams GM, Thomas BP. Optical remote sensing for monitoring flying mosquitoes, gender identification and discussion on species identification. Appl Phys B. 2018; 124: 46. 
26. Costello RA. Effects of environmental and physiological factors on the acoustic behavior of Aedes aegypti (L.) (Diptera: Culicidae) [PhD Thesis]. Winnipeg: University of Manitoba; 1974.

27. Staunton KM, Usher L, Prachar T, Ritchie SA, Snoad N, Johnson BJA. Novel methodology for recording wing beat frequencies of untethered male and female Aedes aegypti. J Am Mosq Control Assoc. 2019; 35(3): 169-77.

28. Villarreal SM, Winokur O, Harrington LC. The impact of temperature and body size on fundamental flight tone variation in the mosquito vector Aedes aegypti (Diptera: Culicidae): implications for acoustic lures. J Med Entomol. 2017; 54(5): 1116-21.

29. Vigoder FM, Ritchie MG, Gibson G, Peixoto AA. Acoustic communication in insect disease vectors. Mem Inst Oswaldo Cruz. 2013; 108(Suppl. 1): 26-33.

30. Lounibos LP, Nishimura N, Conn J, Lourenço-de-Oliveira R. Life history correlates of adult size in the malaria vector Anopheles darlingi. Mem Inst Oswaldo Cruz. 1995; 90(6): 769-74.

31. Stone CM, Tuten HC, Dobson SL. Determinants of male Aedes aegypti and Aedes polynesiensis (Diptera: Culicidae) response to sound: efficacy and considerations for use of sound traps in the Field. J Med Entomol. 2013; 50(4): 723-30.

32. Johnson BJ, Rohde BB, Zeak N, Staunton KM, Prachar T, Ritchie SA. A low-cost, battery-powered acoustic trap for surveilling male Aedes aegypti during rear-and-release operations. PLoS One. 2018; 13(8): e0201709.

33. Potamitis I, Rigakis I. Measuring the fundamental frequency and the harmonic properties of the wingbeat of a large number of mosquitoes in flight using 2D optoacoustic sensors. Appl Acoust. 2016; 109: 54-60.

34. Moreno M, Tong C, Guzmán M, Chuquiyauri R, Llanos-Cuentas A, Rodriguez H, et al. Infection of laboratory-colonized Anopheles darlingi mosquitoes by Plasmodium vivax. Am J Trop Med Hyg. 2014; 90(4): 612-16.

35. Lainhart W, Bickersmith SA, Moreno M, Rios CT, Vinetz JM, Conn JE. Changes in genetic diversity from field to laboratory during colonization of Anopheles darlingi Root (Diptera: Culicidae). Am J Trop Med Hyg. 2015; 93(5): 998-1001.
36. van den Heuvel MJ. The effects of rearing temperature on the wing length, thorax length and ovariole number of the adult mosquito, Aedes aegypti (L.). Trans R. Entomol Soc London. 1963; 115(7): 197-216.

37. John F, Sanford W. An R companion to applied regression. 3rd ed. SAGE: $2018 ; 608$ pp.

38. Core team R. R: a language and environment for statistical computing. Vienna: R Foundation for Statistical Computing; 2018.

39. Ramírez-Sánchez F, Camargo C, Avila FW. Male sexual history influences female fertility and re-mating incidence in the mosquito vector Aedes aegypti (Diptera: Culicidae). J Insect Physiol. 2020; 121: 104019.

40. Arthur BJ, Emr KS, Wyttenbach RA, Hoy RR. Mosquito (Aedes aegypti ) flight tones: frequency, harmonicity, spherical spreading, and phase relationships. J Acoust Soc Am. 2014; 135(2): 933-41.

41. Lardeux F, Quispe V, Tejerina R, Rodríguez R, Torrez L, Bouchité $\mathrm{B}$, et al. Laboratory colonization of Anopheles pseudopunctipennis (Diptera: Culicidae) without forced mating. CR Biol. 2007; 330(8): 571-5.

42. Aldersley A, Pongsiri A, Bunmee K, Kijchalao U, Chittham W, Fansiri T, et al. Too "sexy" for the field? Paired measures of laboratory and semi-field performance highlight variability in the apparent mating fitness of Aedes aegypti transgenic strains. Parasit Vectors. 2019; 12: 357.

43. Cator LJ, Zanti Z. Size, sounds and sex: interactions between body size and harmonic convergence signals determine mating success in Aedes aegypti. Parasit Vectors. 2016; 9: 622.

44. Charlwood JD, Pinto J, Sousa CA, Madsen H, Ferreira C, do Rosario VE. The swarming and mating behaviour of Anopheles gambiae s.s. (Diptera: Culicidae) from São Tomé Island. J Vector Ecol. 2002; 27(2): 178-3.

45. Charlwood JD, Thompson R, Madsen H. Observations on the swarming and mating behaviour of Anopheles funestus from southern Mozambique. Malar J. 2003; 2: 2. 